\title{
Presence of autoantibodies in serum does not impact the occurrence of immune checkpoint inhibitor-induced hepatitis in a prospective cohort of cancer patients
}

\author{
Mette-Triin Purde ${ }^{1} \cdot$ Rebekka Niederer $^{1} \cdot$ Nikolaus B. Wagner $^{2} \cdot$ Stefan Diem $^{1,3,4} \cdot$ Fiamma Berner $^{1}$. \\ Omar Hasan Ali $^{1,5}$. Dorothea Hillmann ${ }^{6}$. Irina Bergamin ${ }^{7} \cdot$ Markus Joerger $^{3} \cdot$ Martin Risch $^{6}$.

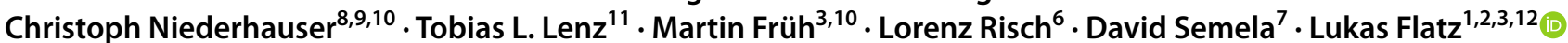

Received: 26 July 2021 / Accepted: 25 November 2021 / Published online: 7 December 2021

(c) The Author(s) 2021

\begin{abstract}
Purpose Immune checkpoint inhibitor (ICI)-induced hepatitis belongs to the frequently occurring immune-related adverse events (irAEs), particularly with the combination therapy involving ipilimumab and nivolumab. However, predisposing factors predicting the occurrence of ICI-induced hepatitis are barely known. We investigated the association of preexisting autoantibodies in the development of ICI-induced hepatitis in a prospective cohort of cancer patients.

Methods Data from a prospective biomarker cohort comprising melanoma and non-small cell lung cancer (NSCLC) patients were used to analyze the incidence of ICI-induced hepatitis, putatively associated factors, and outcome.

Results 40 patients with melanoma and 91 patients with NSCLC received ICI between July 2016 and May 2019. 11 patients developed ICI-induced hepatitis (8.4\%). Prior to treatment, $45.5 \%$ of patients in the hepatitis cohort and $43.8 \%$ of the control cohort showed elevated titers of autoantibodies commonly associated with autoimmune liver diseases $(p=0.82)$. We found two nominally significant associations between the occurrence of ICI-induced hepatitis and HLA alleles associated with autoimmune liver diseases among NSCLC patients. Of note, significantly more patients with ICI-induced hepatitis developed additional irAEs in other organs $(p=0.0001)$. Neither overall nor progression-free survival was affected in the hepatitis group. Conclusion We found nominally significant associations of ICI-induced hepatitis with two HLA alleles. ICI-induced hepatitis showed no correlation with liver-specific autoantibodies, but frequently co-occurred with irAEs affecting other organs. Unlike other irAEs, ICI-induced hepatitis is not associated with a better prognosis.
\end{abstract}

Keywords Autoantibodies · Checkpoint inhibitors · Drug-induced liver injury · Drug-related side effects and adverse reactions

\section{Introduction}

Immune checkpoint inhibitors (ICI) have significantly prolonged the survival of patients with various cancers (Iorgulescu et al. 2018; Massari et al. 2018). ICI are monoclonal antibodies that target inhibitory immune checkpoints on $\mathrm{T}$ cells, thereby reinvigorating their anti-tumor activity (Silva et al. 2018). Currently FDA-approved ICI target either programmed cell death protein 1 (PD-1), its ligand programmed

Lukas Flatz

lukas.flatz@med.uni-tuebingen.de

Extended author information available on the last page of the article death ligand 1 (PD-L1), or cytotoxic T lymphocyte-associated protein 4 (CTLA4).

The clinical use of ICI is curtailed by immune-related adverse events (irAEs) that develop in up to $70 \%$ of all patients (Freeman-Keller et al. 2016; Wang et al. 2017; Owen et al. 2018). Severe irAEs require therapy interruption or discontinuation and systemic immunosuppression, which may inhibit therapeutic efficacy (Brahmer et al. 2018).

ICI-induced hepatitis is relatively common but remains poorly characterized. Studies suggest that it occurs in up to $20 \%$ of patients, depending on the ICI treatment (Nishida and Kudo 2019). ICI-induced hepatitis is usually asymptomatic and detected by elevated liver enzymes (European Association for the Study of the Liver. Electronic address et al. 2019). The histopathological traits of ICI-induced 
hepatitis overlap with both viral and autoimmune hepatitis (Nadeau et al. 2018; Suzman et al. 2018).

Autoimmune liver diseases include autoimmune hepatitis (AIH), primary biliary cholangitis, and primary sclerosing cholangitis, as well as their overlap syndromes. All of these diseases are associated with various autoantibodies and human leukocyte antigen (HLA) alleles (Chen et al. 2019; Lee and Ronnekleiv-Kelly 2019).

The application of ICI in patients with preexisting liver diseases or hepatic dysfunction is controversial due to lacking reports (Kanz et al. 2016; Aizawa and Hokari 2017; Suzman et al. 2018; Kehl et al. 2019; Shah et al. 2019). As of now, there are no validated biomarkers for predicting the development of ICI-induced hepatitis. To better understand the development of ICI-induced hepatitis, we characterized it with clinical and laboratory parameters in a prospective cohort of metastatic cancer patients receiving ICI.

\section{Materials and methods}

\section{Study population}

We established a prospective observational cohort of metastatic melanoma and non-small cell lung cancer (NSCLC) patients that started treatment with ICI (anti-PD-1, antiPD-L1, anti-CTLA4, or combination treatment). The study was conducted at the Kantonsspital St. Gallen, Switzerland. All participants provided written informed consent prior to study inclusion. We used the STROBE cohort checklist when writing our report (von Elm et al. 2014).

Patients were enrolled between July 15, 2016 and May 1, 2019. Follow-up data were collected until October 15, 2019. Patients without ICI-induced hepatitis who received at least five cycles of any ICI therapy were included in the control group. Patients without ICI-induced hepatitis who had received less than five cycles of ICI therapy were excluded from the analysis of putative predictive factors. None of the patients had preexisting autoimmune liver diseases. The frequency of other preexisting autoimmune diseases was comparable in patients with and without ICI-induced hepatitis.

The following clinical information was recorded for all participants: sex, age, preexisting autoimmune diseases, and previous cancer therapy. In case of early dropout or exclusion, timepoint of and reason for leaving the study were recorded. Response to ICI therapy was determined based on the first computed tomography (CT) scan at cycle 4-6 (8-12 weeks) and according to the RECIST criteria version 1.1 (Eisenhauer et al. 2009). The presence and grade of ICIinduced hepatitis were defined according to CTCAE version 5.0 (2017, European Association for the Study of the Liver. Electronic address et al. 2019). The grade was based on liver function tests [LFTs; AST, ALT, bilirubin, and alkaline phosphatase (ALP)], as previously defined (Brahmer et al. 2018). All LFTs were measured from before the first ICI administration until study end or dropout.

Two experienced board-certified gastroenterologists and hepatologists (IB and DS) reviewed all cases independently and determined the likelihood of ICI-induced hepatotoxicity versus an alternative etiology according to well-characterized criteria (Benichou et al. 1993; Fontana et al. 2009). The $\mathrm{R}$ value for liver injury was calculated by dividing the peak serum level of ALT/ULN by that of ALP/ULN (European Association for the Study of the Liver. Electronic address et al. 2019). The type of liver damage was determined from the $\mathrm{R}$ value as hepatocellular $(R>5)$, mixed $(2 \leq R \leq 5)$, or cholestatic $(R<2)$ (European Association for the Study of the Liver. Electronic address et al. 2019).

Furthermore, we recorded the occurrence of irAEs affecting other organs, defined according to the CTCAE version 5.0 (2017, European Association for the Study of the Liver. Electronic address et al. 2019). The onset, duration, and type of systemic immunosuppressive therapy for ICI-induced hepatitis treatment, as well as the presence of metastases and other lesions in the liver (e.g., steatosis; based on ultrasonography and CT scans) were also recorded.

\section{Blood sampling and analyses}

Blood sampling and processing was performed at every visit according to the treating physician's instructions. In the hepatitis group, patient sera were analyzed at three timepoints: before first ICI application, at the onset of ICIinduced hepatitis (elevated LFTs), and 2 months later. For control patients without ICI-induced hepatitis, we analyzed pre-therapy serum samples.

We measured autoantibodies that have been associated with autoimmune liver diseases (listed in Supplementary Table 1). The titer and pattern of anti-nuclear (ANA) and anti-cytoplasmatic antibodies, as well as the titers of antismooth muscle, anti-actin, anti-mitochondrial, and antiliver-kidney microsomal antibodies were determined by the indirect immunofluorescence test on the IF Sprinter (Euroimmun, Lübeck, Germany) according to the manufacturer's instructions. ANA patterns were determined in adherence to the nomenclature of the International Consensus on ANA Patterns (Chan et al. 2015). Serum concentration of perinuclear anti-neutrophil cytoplasmatic antibodies was measured by fluorescent enzyme immunoassay on the Phadia 250 (Thermo Fisher Scientific, Waltham, MA). The following autoantibodies were detected via immunoblot analysis using the EUROLINE Autoimmune Liver Diseases Profile (Euroimmun, Lübeck, Germany): AMA-M2, M2-3E (BPO), Sp100, PML, gp210, LKM1, LC1, SLA/LP, 
and Ro-52. Total serum IgG was measured by turbidimetry on the Optilite (The Binding Site, Birmingham, UK).

Sera from hepatitis patients were screened for infection with hepatitis $\mathrm{A}, \mathrm{B}, \mathrm{C}$, and $\mathrm{E}$ viruses (HAV, $\mathrm{HBV}, \mathrm{HCV}$, and $\mathrm{HEV}$, respectively). HAV IgG and IgM, HCV IgG, HBV surface antigen and antibodies against $\mathrm{HBV}$ core antigen were detected by electrochemiluminescence immunoassay on the Cobas 6000 (Roche Diagnostics, Rotkreuz, Switzerland). $\mathrm{HCV}$ antigen was detected by chemiluminescent microparticle immunoassay on the Architect i2000SR (Abbott Laboratories, Lake Bluff, IL). HEV IgG and IgM were measured using enzyme-linked immunosorbent assays on the DSX (Dynex Technologies, Chantilly, VA). HEV PCR was performed on the Cobas 8800 (Roche Diagnostics, Rotkreuz, Switzerland). The inter-assay coefficients of variation for relevant analyses are provided in Supplementary Table 2.

\section{HLA typing}

DNA for HLA haplotyping was isolated from peripheral blood mononuclear cells. All patients were genotyped for HLA loci A, B, C, DPB1, DQB1, and DRB1. Genotyping was performed using next-generation sequencing at six-digit resolution $(4 \times$ High Resolution Typing, HistoGenetics, Ossining, NY).

\section{Statistical analysis}

Continuous variables were compared using unpaired twotailed $t$ test with Welch's correction. Proportions were compared using Chi-squared test with or without Yates' correction, as appropriate. Patients whose response to ICI therapy at first CT scan was unknown were excluded from analyses pertaining to therapy response. Survival curves were compared by log-rank test. Patients who were alive or progression-free at the end of the follow-up period were censored in overall and progression-free survival analysis, respectively. Patients lost to follow-up were censored at the timepoint of last contact. To correct for multiple comparisons of survival curves, $p$ values were adjusted using the Holm-Š́ídák method. All statistical analyses were performed using Prism version 8.1.2 (GraphPad Software, San Diego, CA).

\section{Results}

\section{Patient characteristics}

135 patients were screened for study inclusion. Four patients were excluded: three because they never began ICI therapy and one due to change of therapy after one cycle of ICI treatment (Supplementary Fig. 1). We included 131 patients, of whom 11 developed ICI-induced hepatitis (8.4\%). The average onset of ICI-induced hepatitis was 12.8 weeks (range 2.7-48.9 weeks) after therapy initiation. 73 patients fulfilled the inclusion criteria for the control group. The mean followup time was 53.4 weeks (1-153 weeks). Detailed clinical characteristics of the hepatitis patients are shown in Table 1.

Five patients (45.5\%) presented with hepatitis of grade 3 or higher (Table 1). There were two cases of hepatocellular damage ( $R$ value $=15.47$ and 23.47, respectively), while the other patients had liver damage of cholestatic or mixed type.

ICI therapy was stopped in nine patients and restarted in three of them after 2-7 weeks. In one patient, LFTs increased after another ICI dose and therapy was discontinued. Six patients received systemic steroid treatment, which led to the normalization of their LFTs after 1 week to several months (Table 1). In the remaining five patients, LFTs normalized without steroid use. No other immunosuppressants were given.

\section{Viral hepatitis}

No evidence of an active infection with HAV, HBV, HCV, or HEV was found among the ICI-induced hepatitis group (Supplementary Table 3). Elevated HAV and HEV IgG indicated past infection with or vaccination against hepatitis A and/or E in five patients. Active infection was ruled out via negative IgM and viral load PCR.

\section{Autoantibodies associated with autoimmune liver diseases}

We analyzed patient sera for the presence of autoantibodies associated with autoimmune liver diseases (listed in Supplementary Table 1). In 7/11 (63.6\%) patients with ICI-induced hepatitis, we measured at least one elevated autoantibody (Supplementary Table 4). 5/11 (45.5\%) hepatitis patients and 32/73 (43.8\%) control patients had an undoubtedly positive titer of at least one autoantibody ( $p=0.82$; Table 2). When borderline positive titers were included, there was a higher proportion of patients with autoantibodies in the control group ( $82.2 \%$ vs $63.6 \%, p=0.31)$. We observed no significant changes in the titers over the course of ICI treatment (Supplementary Table 4). We did not find statistically significant differences in ANA patterns or single antibodies between the two groups (Supplementary Table 5).

\section{HLA alleles}

To assess the association between HLA and ICI-induced hepatitis, we screened hepatitis and control patients for the HLA alleles that are associated with a predisposition to or protection from autoimmune liver diseases (Supplementary Table 6). Among patients with NSCLC, two alleles were nominally significantly associated with the risk of 


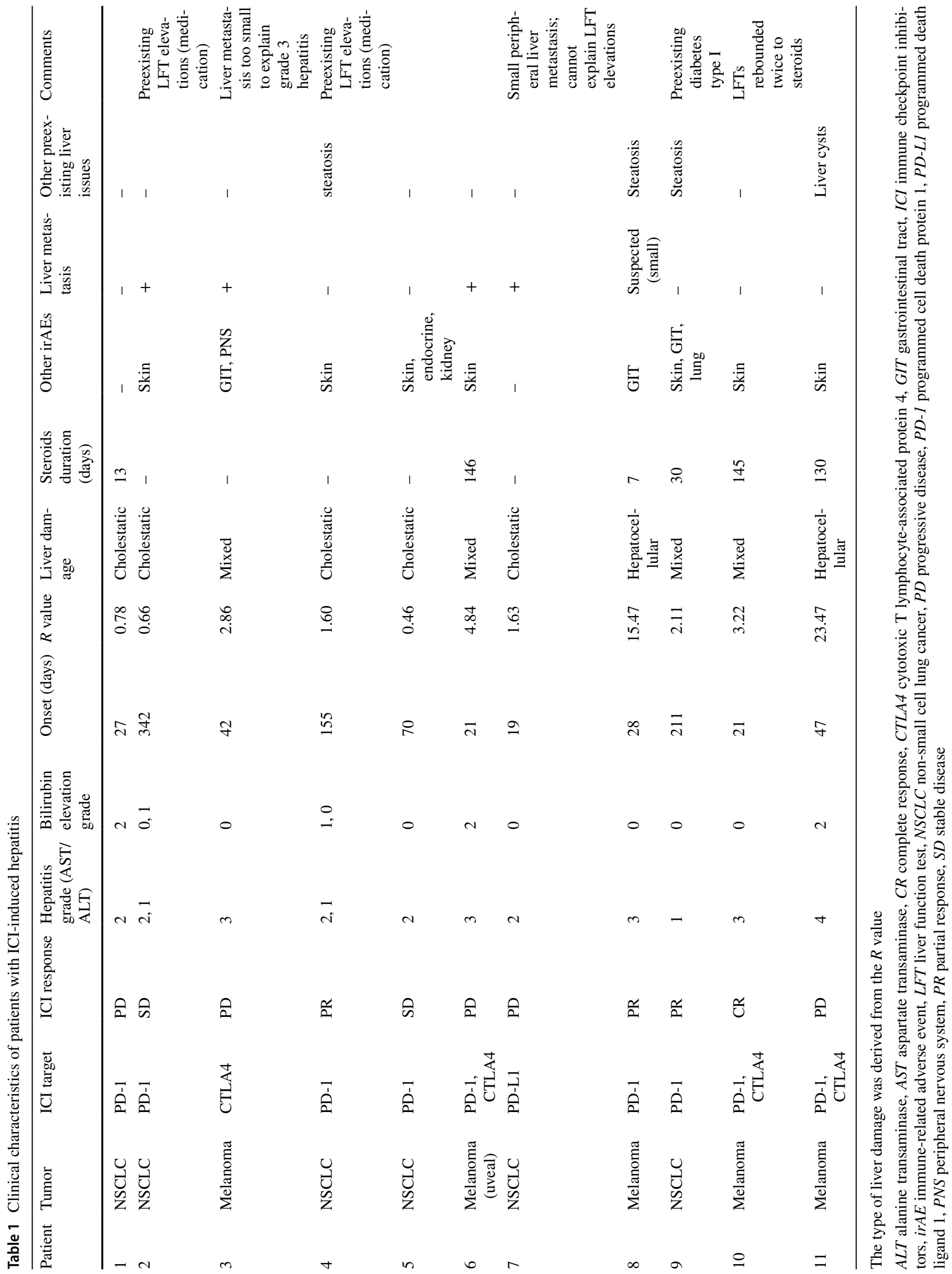


Table 2 Positive titers of liver autoantibodies in patients with and without ICI-induced hepatitis

\begin{tabular}{lll}
\hline & Number & Proportion \\
\hline Hepatitis $(N=11)$ & 5 & $45.5 \%$ \\
No hepatitis $(N=73)$ & 32 & $43.8 \%$ \\
$p$ value & 0.82 & \\
\hline
\end{tabular}

ICI immune checkpoint inhibitors

developing ICI-induced hepatitis: DRB $1 * 04: 01(p=0.037)$ and the haplotype DRB $1 * 15: 01-\mathrm{DQB} 1 * 06: 02(p=0.04)$ (Fig. 1). However, the statistical significance of those associations was absent in the whole patient population and after correcting for multiple comparisons, which indicates the need for studies in larger cohorts to confirm the finding.

\section{Survival and therapy response}

In the group with ICI-induced hepatitis, there was one death during the study for medical reasons unrelated to the liver. There were no deaths caused by liver failure or liver metastasis.

IrAEs affecting the skin are well-known adverse effects of ICI therapy and associated with improved survival
(Hasan Ali et al. 2016). 42 patients (32.1\% of all patients) experienced skin irAEs in this study. We investigated the associations of overall survival (OS) and progression-free survival (PFS) with ICI-induced hepatitis and skin irAEs (Fig. 2). Only skin irAEs were associated with longer OS and PFS ( $p=0.0003$ for both). In contrast, there were no significant differences between patients with ICI-induced hepatitis and the rest of the cohort (OS, $p=0.23$; PFS, $p=0.35$ ). The patients with ICI-induced hepatitis did not respond more often to ICI therapy than the control group ( $36.4 \%$ vs $41.8 \%, p=0.99$ ). The proportion of patients with progressive disease was higher among patients with ICI-induced hepatitis $(45.5 \%$ vs $13.4 \%, p=0.03)$.

The patients with and without ICI-induced hepatitis did not differ significantly with regards to age, sex ratio, or prior exposure to ICI therapy (Table 3). One patient in the hepatitis group and three control patients had preexisting autoimmune diseases (type I diabetes mellitus, multiple sclerosis, Guillain-Barré syndrome, and ulcerative colitis). The proportion of patients with autoimmune diseases did not differ between the groups. However, we found a highly significant difference in the development of multiple irAEs: 9/11 patients in the ICI-induced hepatitis group (81.8\%) developed additional irAEs, compared with 15/73 controls with multiple irAEs $(20.5 \% ; p=0.0001)$.
Fig. 1 Proportion of HLA allele DRB $1 * 04: 01$ and haplotype DRB1*15:01-DQB $1 * 06: 02$ in patients with ICI-induced hepatitis and controls among the whole cohort (a) and among patients with NSCLC (b). These alleles are associated with autoimmune liver diseases and exhibited a significant association with ICI-induced hepatitis among NSCLC patients in this cohort. ICI immune checkpoint inhibitors, NSCLC non-small cell lung cancer

\section{a} $\mathrm{ICl}$-induced hepatitis

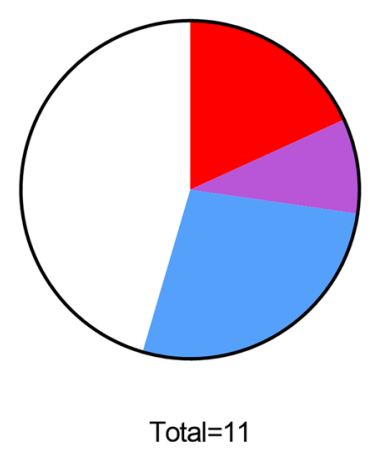

b $\mathrm{ICl}$-induced hepatitis

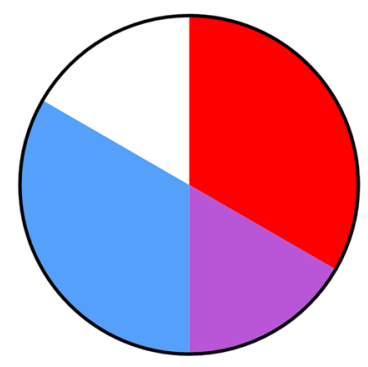

Total $=6$
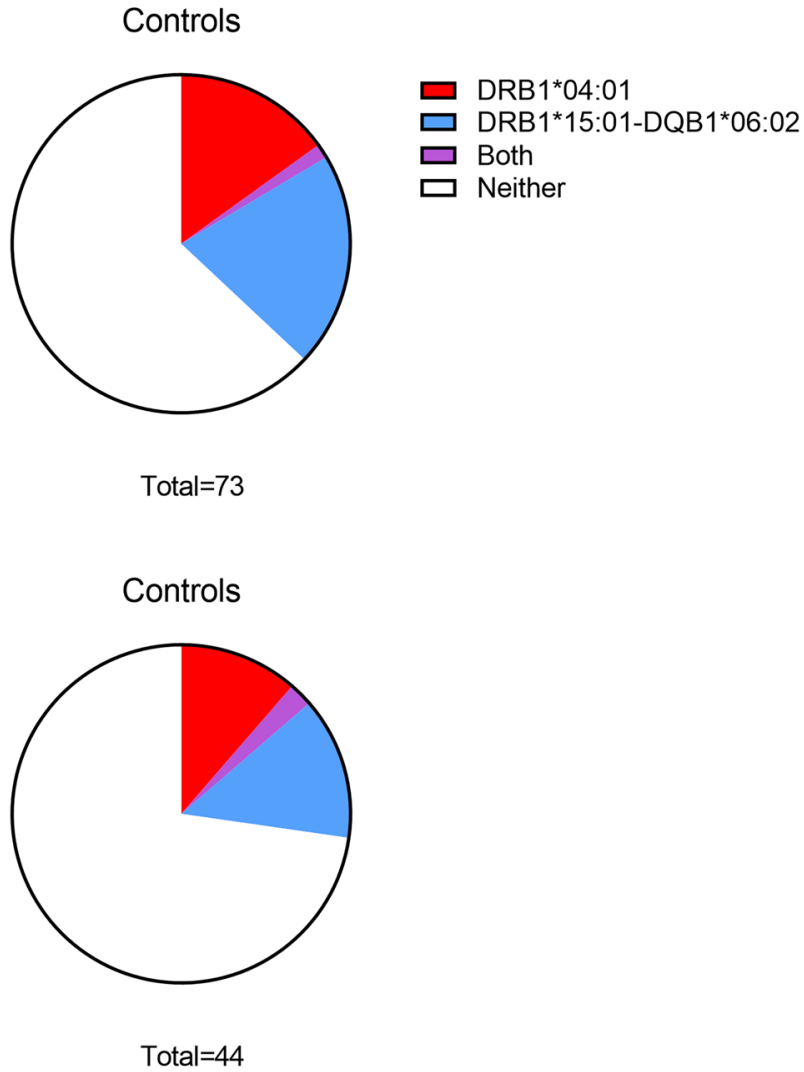
Fig. 2 Overall (a) and progression-free survival (b) of patients with ICI-induced hepatitis and skin irAEs compared with the rest of the cohort. $d$ days, $I C I$ immune checkpoint inhibitors, irAE immune-related adverse event
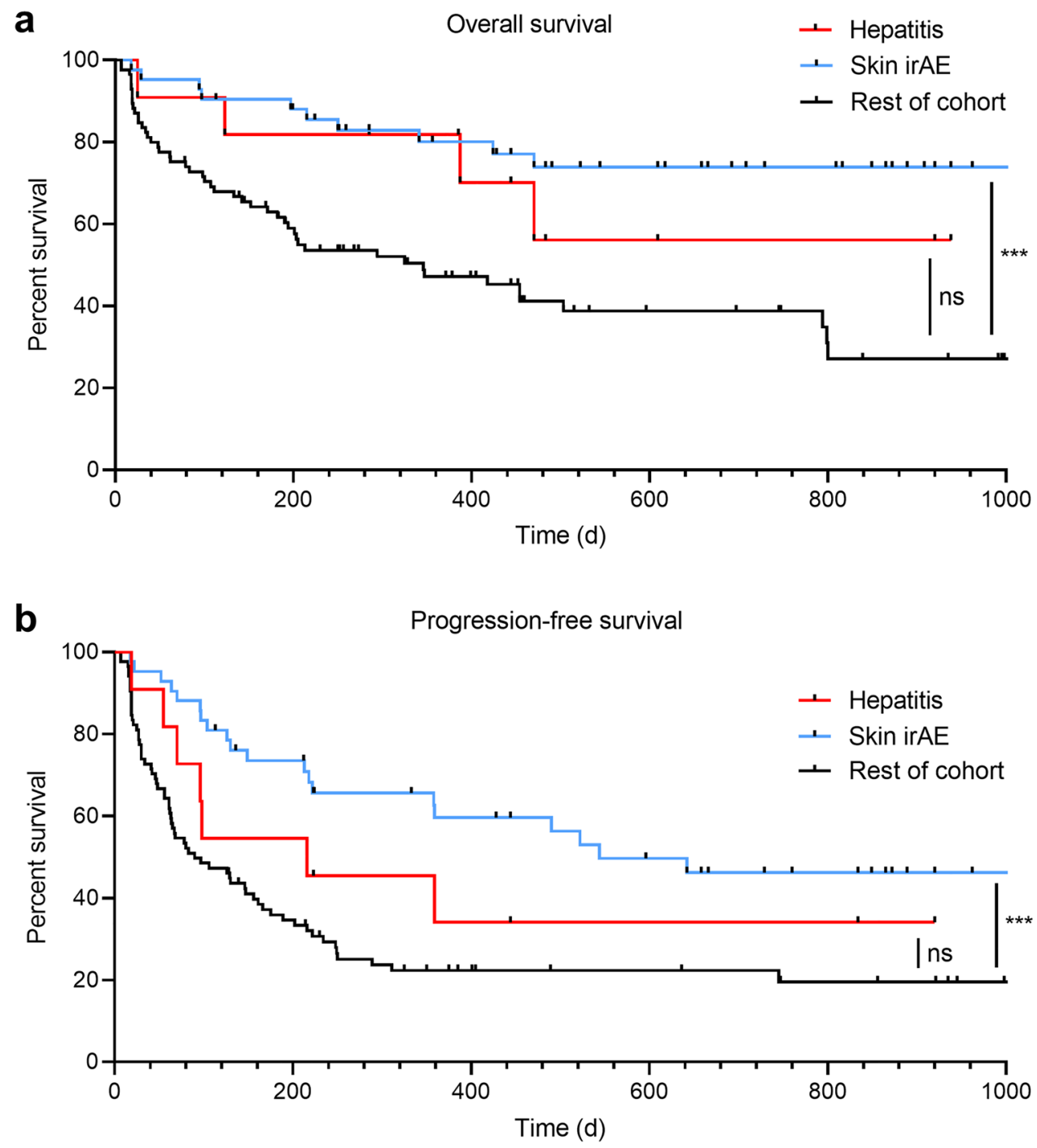

Table 3 Comparison of clinical characteristics of patients with and without ICI-induced hepatitis

\begin{tabular}{llll}
\hline & $\begin{array}{l}\text { ICI-induced hepa- } \\
\text { titis } \\
N=11\end{array}$ & $\begin{array}{l}\text { Control group } \\
N=73\end{array}$ & $p$ value \\
& & & \\
\hline $\begin{array}{l}\text { Age, median (range) } \\
\text { Sex, M:F }\end{array}$ & $51(41-73)$ & $67(47-86)$ & 0.13 \\
$\begin{array}{l}\text { Prior ICI therapy, } \\
N(\%)\end{array}$ & $2(18.2 \%)$ & $40: 33$ & 0.80 \\
$\begin{array}{l}\text { Preexisting autoim- } \\
\text { mune disease, } N \\
(\%)\end{array}$ & $1(9.1 \%)$ & $3(4.1 \%)$ & 0.85 \\
$\begin{array}{l}\text { Multiple irAEs, } N \\
(\%)\end{array}$ & $9(81.8 \%)$ & & \\
\hline
\end{tabular}

Bold value indicates statistically significant $p$ value

ICI immune checkpoint inhibitor, irAE immune-related adverse event

\section{Discussion}

This study systematically investigated the development of ICI-induced hepatitis during ICI therapy and their associations with patient characteristics. To this end, we established a prospective cohort of metastatic melanoma and NSCLC patients treated with ICI. Viral hepatitis was excluded in all patients with elevated LFTs. The incidence and average onset of ICI-induced hepatitis were comparable with previous publications (Karamchandani and Chetty 2018; Suzman et al. 2018).

It is known that liver metastases can increase LFTs (Beck et al. 1979; Cao and Wang 2012). In a recent study of liver injury in patients receiving pembrolizumab, pretreatment liver metastases were identified as the only independent predictor of LFT elevations (Tsung et al. 2019). In our study, two patients (Patients 6 and 8) had LFT elevations that may have occurred due to a liver metastasis (Table 1). Two other patients ( 2 and 4 ) had elevated LFTs 
already prior to the start of ICI therapy, which we consider likely to be caused by other concomitant drugs.

We measured the titers of autoantibodies associated with classical autoimmune liver diseases in patients developing ICI-induced hepatitis. The data show no statistically significant differences in overall titers or specific autoantibodies compared with control patients. While other authors have shown associations between autoantibodies and the occurrence of ICI-induced skin toxicity, hypophysitis, and pneumonitis (Ali et al. 2019; Tahir et al. 2019), we did not find such an association with ICI-induced hepatitis. Our data are in line with the previous findings that show high levels of autoantibodies in healthy volunteers (Okamoto et al. 2004; Slight-Webb et al. 2016) and cancer patients (Anderson et al. 2015; Nisihara et al. 2018). Furthermore, the previous reports similarly show no elevated titers of liver-specific autoantibodies in patients with ICI-induced hepatitis (Johncilla et al. 2015; De Martin et al. 2018; Zen and Yeh 2018). Therefore, our data indicate that liver autoantibodies are not suitable predictors for the development of ICI-induced hepatitis. Moreover, this suggests that the pathogenesis of ICI-induced hepatitis may differ from that of classical autoimmune hepatitis. This also indicates that patients with preexisting liver autoantibodies do not have an increased risk of developing hepatitis during ICI treatment.

We studied whether HLA alleles associated with autoimmune liver diseases are also associated with ICI-induced hepatitis. Interestingly, we detected two alleles with nominal statistical significance among NSCLC patients. However, the significance disappeared upon correction for multiple comparisons, possibly because of the small sample size. The effect may have been too weak to detect at this study power. This highlights the necessity of conducting larger studies to investigate the role of HLA alleles in the development of ICI-induced hepatitis.

The occurrence of irAEs has been associated with better therapy response and prolonged survival (Sato et al. 2018; Okada et al. 2019). We observed this association for patients with skin irAEs. Conversely, the development of ICI-induced hepatitis did not correlate with OS or PFS and even showed an association with worse therapy response. This is in line with a recent study showing a trend toward favorable OS and PFS only for patients with ICI-induced hepatitis of grade 3-4 (Biewenga et al. 2021).

We also found a highly significant co-occurrence of ICIinduced hepatitis and other irAEs. However, patients with probable ICI-induced hepatitis have shown better survival than patients with liver injury due to other causes (Tsung et al. 2019) and a recent study showed improved overall survival in melanoma patients who developed elevated levels of gamma-glutamyl transferase during ICI treatment (Winter et al. 2021). It is possible that improved survival among the patients with ICI-induced hepatitis could not be detected in this study because of the small sample size.

Our study was limited by the small sample size of the ICI-induced hepatitis cohort, which may restrict statistical power. Nevertheless, we were able to show nominally significant associations between ICI-induced hepatitis and two HLA alleles, as well as a highly significant co-occurrence of ICI-induced hepatitis with other irAEs. Investigating these findings in larger cohorts may reveal additional associations. In a follow-up study, it would be beneficial to obtain liver biopsies from all patients with ICI-induced hepatitis to also include histological findings.

In conclusion, this study characterized ICI-induced hepatitis in detail in a prospective cohort of cancer patients receiving ICI treatment. Active hepatitis virus infection could be excluded in all patients with elevated LFTs. We found no associations between ICI-induced hepatitis occurrence and markers of classical autoimmune liver disease, suggesting a mechanistic difference in pathogenesis and no increased risk of ICI-induced hepatitis for patients with preexisting liver autoantibodies. In contrast, we discovered a nominally significant association between ICI-induced hepatitis and two HLA alleles in NSCLC patients. These data emphasize the role of genetic background as a major predictor of irAE development. Unlike skin irAEs, ICI-induced hepatitis was not associated with improved therapy response or survival, but was associated with additional irAEs. This implies that liver involvement may signal the presence of other irAEs.

Supplementary Information The online version contains supplementary material available at https://doi.org/10.1007/s00432-021-03870-6.

Acknowledgements We wholeheartedly thank all participating patients, doctors, and nurses who made this study possible. Part of this study has previously been presented at the SGG-SGVC-SASL annual congress of 2019 in Interlaken as an oral presentation.

Author contributions LF designed the project with input from DS, SD, MF, MJ, and IB. Patient enrolment and data collection were done by LF, SD, DS, RN, and OHA. Blood samples were processed by RN, FB, OHA, and MTP. LR, DH, MR, and CN conducted the laboratory analyses. Data analysis was performed by MTP and TLL, with input from LF, NBW, and DS. MTP and LF drafted the initial manuscript. All authors participated in reviewing of the manuscript and approved the final version.

Funding Open Access funding enabled and organized by Projekt DEAL. This project was supported by the Swiss National Science Foundation grant PP00P3_157448 and the Swiss Cancer League grant KLS-4409-02-2018 (both to LF). Study coordination of this patient cohort was supported by a grant from the Forschungsförderung of the Kantonsspital St. Gallen.

Availability of data and materials The datasets used in this study are available from the corresponding author on reasonable request. 
Code availability Not applicable.

\section{Declarations}

Conflict of interest NBW reported an advisory role for Sanofi and travel support from AbbVie and Amgen. MF reported grants from Bristol-Myers Squibb outside of the submitted work and advisory role for AstraZeneca, Merck Sharp \& Dohme, Roche, Bristol-Myers Squibb, and Boehringer Ingelheim. LF reported grants outside of the submitted work from Hookipa Pharma and Novartis Foundation as well as an advisory role for Novartis, Sanofi, and Bristol-Myers Squibb. All other authors reported no competing interests.

Ethics approval The study was approved by the Ethics Committee of Eastern Switzerland (project number EKOS 2016-00998). The study was conducted in accordance with the Declaration of Helsinki.

Consent to participate Written informed consent was obtained from all participants prior to study enrolment.

Consent for publication Written consent for publication of data was obtained from all participants prior to study enrolment.

Open Access This article is licensed under a Creative Commons Attribution 4.0 International License, which permits use, sharing, adaptation, distribution and reproduction in any medium or format, as long as you give appropriate credit to the original author(s) and the source, provide a link to the Creative Commons licence, and indicate if changes were made. The images or other third party material in this article are included in the article's Creative Commons licence, unless indicated otherwise in a credit line to the material. If material is not included in the article's Creative Commons licence and your intended use is not permitted by statutory regulation or exceeds the permitted use, you will need to obtain permission directly from the copyright holder. To view a copy of this licence, visit http://creativecommons.org/licenses/by/4.0/.

\section{References}

(2017) "Common Terminology Criteria for Adverse Events (CTCAE) Version 5.0.” 2019, from https://ctep.cancer.gov/protocoldevelop ment/electronic_applications/docs/CTCAE_v5_Quick_Refer ence_5x7.pdf

Aizawa Y, Hokari A (2017) Autoimmune hepatitis: current challenges and future prospects. Clin Exp Gastroenterol 10:9-18. https://doi. org/10.2147/CEG.S101440

Ali OH, Bomze D, Ring S et al (2019) BP180-specific IgG is associated with skin adverse events, therapy response and overall survival in non-small cell lung cancer patients treated with checkpoint inhibitors. J Am Acad Dermatol. https://doi.org/10.1016/j.jaad. 2019.08.045

Anderson KS, Cramer DW, Sibani S et al (2015) Autoantibody signature for the serologic detection of ovarian cancer. J Proteome Res 14(1):578-586. https://doi.org/10.1021/pr500908n

Beck PR, Belfield A, Spooner RJ, Blumgart LH, Wood CB (1979) Serum enzymes in colorectal cancer. Cancer 43(5):1772-1776. https://doi.org/10.1002/1097-0142(197905)43:5\%3c1772::aidcncr2820430529\%3e3.0.co;2-y

Benichou C, Danan G, Flahault A (1993) Causality assessment of adverse reactions to drugs-II. An original model for validation of drug causality assessment methods: case reports with positive rechallenge. J Clin Epidemiol 46(11):1331-1336. https://doi.org/ 10.1016/0895-4356(93)90102-7
Biewenga M, van der Kooij MK, Wouters M et al (2021) Checkpoint inhibitor induced hepatitis and the relation with liver metastasis and outcome in advanced melanoma patients. Hepatol Int 15(2):510-519. https://doi.org/10.1007/s12072-021-10151-4

Brahmer JR, Lacchetti C, Schneider BJ et al (2018) Management of immune-related adverse events in patients treated with immune checkpoint inhibitor therapy: American society of clinical oncology clinical practice guideline. J Clin Oncol 36(17):1714-1768. https://doi.org/10.1200/JCO.2017.77.6385

Cao R, Wang LP (2012) Serological diagnosis of liver metastasis in patients with breast cancer. Cancer Biol Med 9(1):57-62. https:// doi.org/10.3969/j.issn.2095-3941.2012.01.011

Chan EK, Damoiseaux J, Carballo OG et al (2015) Report of the first international consensus on standardized nomenclature of antinuclear antibody HEp-2 cell patterns 2014-2015. Front Immunol 6:412. https://doi.org/10.3389/fimmu.2015.00412

Chen RL, Wang QX, Ma X (2019) Precision medicine for autoimmune hepatitis. J Dig Dis 20(7):331-337. https://doi.org/10. 1111/1751-2980.12786

De Martin E, Michot JM, Papouin B et al (2018) Characterization of liver injury induced by cancer immunotherapy using immune checkpoint inhibitors. J Hepatol 68(6):1181-1190. https://doi. org/10.1016/j.jhep.2018.01.033

Eisenhauer EA, Therasse P, Bogaerts J et al (2009) New response evaluation criteria in solid tumours: revised RECIST guideline (version 1.1). Eur J Cancer 45(2):228-247. https://doi.org/10. 1016/j.ejca.2008.10.026

European Association for the Study of the Liver. Electronic address eee, Clinical Practice Guideline Panel C, Panel m and representative EGB (2019) EASL clinical practice guidelines: druginduced liver injury. J Hepatol 70(6):1222-1261. https://doi.org/ 10.1016/j.jhep.2019.02.014

Fontana RJ, Watkins PB, Bonkovsky HL et al (2009) Drug-Induced Liver Injury Network (DILIN) prospective study: rationale, design and conduct. Drug Saf 32(1):55-68. https://doi.org/10. 2165/00002018-200932010-00005

Freeman-Keller M, Kim Y, Cronin H, Richards A, Gibney G, Weber JS (2016) Nivolumab in resected and unresectable metastatic melanoma: characteristics of immune-related adverse events and association with outcomes. Clin Cancer Res 22(4):886-894. https://doi.org/10.1158/1078-0432.CCR-15-1136

Hasan Ali O, Diem S, Markert E et al (2016) Characterization of nivolumab-associated skin reactions in patients with metastatic non-small cell lung cancer. Oncoimmunology 5(11):e1231292. https://doi.org/10.1080/2162402X.2016.1231292

Iorgulescu JB, Harary M, Zogg CK et al (2018) Improved riskadjusted survival for melanoma brain metastases in the era of checkpoint blockade immunotherapies: results from a national cohort. Cancer Immunol Res 6(9):1039-1045. https://doi.org/ 10.1158/2326-6066.CIR-18-0067

Johncilla M, Misdraji J, Pratt DS et al (2015) Ipilimumab-associated hepatitis: clinicopathologic characterization in a series of 11 cases. Am J Surg Pathol 39(8):1075-1084. https://doi.org/10. 1097/PAS.0000000000000453

Kanz BA, Pollack MH, Johnpulle R et al (2016) Safety and efficacy of anti-PD-1 in patients with baseline cardiac, renal, or hepatic dysfunction. J Immunother Cancer 4:60. https://doi.org/10. 1186/s40425-016-0166-5

Karamchandani DM, Chetty R (2018) Immune checkpoint inhibitorinduced gastrointestinal and hepatic injury: pathologists' perspective. J Clin Pathol 71(8):665-671. https://doi.org/10.1136/ jclinpath-2018-205143

Kehl KL, Yang S, Awad MM, Palmer N, Kohane IS, Schrag D (2019) Pre-existing autoimmune disease and the risk of immune-related adverse events among patients receiving checkpoint inhibitors 
for cancer. Cancer Immunol Immunother 68(6):917-926. https://doi.org/10.1007/s00262-019-02321-z

Lee CW, Ronnekleiv-Kelly S (2019) Autoimmune diseases of the biliary tract: a review. Surg Clin North Am 99(2):185-201. https:// doi.org/10.1016/j.suc.2018.11.003

Massari F, Di Nunno V, Cubelli M et al (2018) Immune checkpoint inhibitors for metastatic bladder cancer. Cancer Treat Rev 64:11-20. https://doi.org/10.1016/j.ctrv.2017.12.007

Nadeau BA, Fecher LA, Owens SR, Razumilava N (2018) Liver toxicity with cancer checkpoint inhibitor therapy. Semin Liver Dis 38(4):366-378. https://doi.org/10.1055/s-0038-1667358

Nishida N, Kudo M (2019) Liver damage related to immune checkpoint inhibitors. Hepatol Int 13(3):248-252. https://doi.org/10. 1007/s12072-018-9921-7

Nisihara R, Machoski MCC, Neppel A, Maestri CA, Messias-Reason I, Skare TL (2018) Anti-nuclear antibodies in patients with breast cancer. Clin Exp Immunol 193(2):178-182. https://doi. org/10.1111/cei.13136

Okada N, Kawazoe H, Takechi K et al (2019) Association between immune-related adverse events and clinical efficacy in patients with melanoma treated with nivolumab: a multicenter retrospective study. Clin Ther 41(1):59-67. https://doi.org/10.1016/j.clint hera.2018.11.004

Okamoto M, Ogawa Y, Watanabe A et al (2004) Autoantibodies to DFS70/LEDGF are increased in alopecia areata patients. J Autoimmun 23(3):257-266. https://doi.org/10.1016/j.jaut.2004.07.004

Owen DH, Burkart J, Patel S et al (2018) 1772PImmune related adverse events across cancer types: incidence, risk factors and survival. Ann Oncol. https://doi.org/10.1093/annonc/mdy300.088

Sato K, Akamatsu H, Murakami E et al (2018) Correlation between immune-related adverse events and efficacy in non-small cell lung cancer treated with nivolumab. Lung Cancer 115:71-74. https:// doi.org/10.1016/j.lungcan.2017.11.019

Shah NJ, Al-Shbool G, Blackburn M et al (2019) Safety and efficacy of immune checkpoint inhibitors (ICIs) in cancer patients with HIV, hepatitis B, or hepatitis C viral infection. J Immunother Cancer 7(1):353. https://doi.org/10.1186/s40425-019-0771-1

Silva IPD, Batten M, Long GV (2018) Reinvigorating tumour-infiltrating lymphocytes from checkpoint inhibitor resistant melanomas. Br J Cancer 119(6):661-662. https://doi.org/10.1038/ s41416-018-0218-3
Slight-Webb S, Lu R, Ritterhouse LL et al (2016) Autoantibody-positive healthy individuals display unique immune profiles that may regulate autoimmunity. Arthritis Rheumatol 68(10):2492-2502. https://doi.org/10.1002/art.39706

Suzman DL, Pelosof L, Rosenberg A, Avigan MI (2018) Hepatotoxicity of immune checkpoint inhibitors: an evolving picture of risk associated with a vital class of immunotherapy agents. Liver Int 38(6):976-987. https://doi.org/10.1111/liv.13746

Tahir SA, Gao J, Miura Y et al (2019) Autoimmune antibodies correlate with immune checkpoint therapy-induced toxicities. Proc Natl Acad Sci U S A 116(44):22246-22251. https://doi.org/10. 1073/pnas. 1908079116

Tsung I, Dolan R, Lao CD et al (2019) Liver injury is most commonly due to hepatic metastases rather than drug hepatotoxicity during pembrolizumab immunotherapy. Aliment Pharmacol Ther 50(7):800-808. https://doi.org/10.1111/apt.15413

von Elm E, Altman DG, Egger M et al (2014) The strengthening the reporting of observational studies in epidemiology (STROBE) Statement: guidelines for reporting observational studies. Int $\mathbf{J}$ Surg 12(12):1495-1499. https://doi.org/10.1016/j.ijsu.2014.07. 013

Wang PF, Chen Y, Song SY et al (2017) Immune-related adverse events associated with anti-PD-1/PD-L1 treatment for malignancies: a meta-analysis. Front Pharmacol 8:730. https://doi.org/10.3389/ fphar.2017.00730

Winter J, Lenders MM, Gassenmaier M et al (2021) Prognostic role of gamma-glutamyl transferase in metastatic melanoma patients treated with immune checkpoint inhibitors. Cancer Immunol Immunother 70(4):1089-1099. https://doi.org/10.1007/ s00262-020-02768-5

Zen Y, Yeh MM (2018) Hepatotoxicity of immune checkpoint inhibitors: a histology study of seven cases in comparison with autoimmune hepatitis and idiosyncratic drug-induced liver injury. Mod Pathol 31(6):965-973. https://doi.org/10.1038/s41379-018-0013-y

Publisher's Note Springer Nature remains neutral with regard to jurisdictional claims in published maps and institutional affiliations.

\section{Authors and Affiliations}

\section{Mette-Triin Purde ${ }^{1} \cdot$ Rebekka Niederer $^{1} \cdot$ Nikolaus B. Wagner $^{2} \cdot$ Stefan Diem $^{1,3,4} \cdot$ Fiamma Berner $^{1}$. Omar Hasan $\mathrm{Ali}^{1,5}$. Dorothea Hillmann ${ }^{6}$. Irina Bergamin ${ }^{7}$. Markus Joerger ${ }^{3}$. Martin Risch ${ }^{6}$.

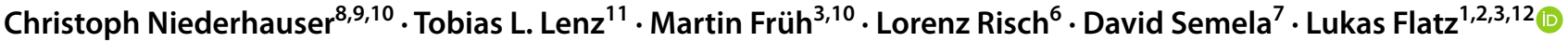

\author{
Mette-Triin Purde \\ mette-triin.purde@kssg.ch \\ Rebekka Niederer \\ rebekka.niederer@kssg.ch \\ Nikolaus B. Wagner \\ nikolaus.wagner@kssg.ch \\ Stefan Diem \\ stefan.diem@kssg.ch \\ Fiamma Berner \\ fiamma.berner@kssg.ch \\ Omar Hasan Ali \\ omar.hasanali@kssg.ch
}

\author{
Dorothea Hillmann \\ dorothea.hillmann@ risch.ch \\ Irina Bergamin \\ irina.bergamin@kssg.ch \\ Markus Joerger \\ markus.joerger@kssg.ch
}

Martin Risch

martin.risch@risch.ch

Christoph Niederhauser

christoph.niederhauser@itransfusion.ch

Tobias L. Lenz

lenz@evolbio.mpg.de 
Martin Früh

martin.frueh@kssg.ch

Lorenz Risch

lorenz.risch@risch.ch

David Semela

david.semela@kssg.ch

1 Institute of Immunobiology, Kantonsspital St. Gallen, Rorschacher Strasse 95, 9007 St. Gallen, Switzerland

2 Department of Dermatology, Venereology and Allergology, Kantonsspital St. Gallen, Rorschacher Strasse 95, 9007 St. Gallen, Switzerland

3 Department of Oncology and Hematology, Kantonsspital St. Gallen, Rorschacher Strasse 95, 9007 St. Gallen, Switzerland

4 Department of Oncology and Hematology, Hospital of Grabs, Spitalstrasse 44, 9472 Grabs, Switzerland

5 Department of Dermatology, University Hospital Zurich, Rämistrasse 100, 8091 Zurich, Switzerland
6 Labormedizinisches Zentrum Dr Risch Ostschweiz AG, Brauerstrasse 95, 9016 St. Gallen, Switzerland

7 Department of Gastroenterology and Hepatology, Kantonsspital St. Gallen, Rorschacher Strasse 95, 9007 St. Gallen, Switzerland

8 Interregional Blood Transfusion SRC, Murtenstrasse 137A, 3008 Bern, Switzerland

9 University of Lausanne, 1015 Lausanne, Switzerland

10 University of Bern, Hochschulstrasse 6, 3012 Bern, Switzerland

11 Research Group for Evolutionary Immunogenomics, Max Planck Institute for Evolutionary Biology, August-Thienemann-Straße 2, 24306 Plön, Germany

12 Department of Dermatology, University Hospital Tübingen, 72016 Tübingen, Germany 